\title{
A Closed-Form Observer for the 3D Inductionless MHD and Navier-Stokes Channel Flow
}

\author{
Rafael Vazquez, Eugenio Schuster and Miroslav Krstic
}

\begin{abstract}
We present a PDE observer that estimates the velocity, pressure, electric potential and current fields in a magnetohydrodynamic (MHD) channel flow, also known as Hartmann flow. This flow is characterized by an electrically conducting fluid moving between parallel plates in the presence of an externally imposed transverse magnetic field. The system is described by the inductionless MHD equations, a combination of the Navier-Stokes equations and a Poisson equation for the electric potential under the so-called MHD approximation in a low magnetic Reynolds number regime. Our observer consists of a copy of the linearized MHD equations, combined with linear injection of output estimation error, with observer gains designed using backstepping. Pressure, skin friction and current measurements from one of the walls are used for output injection. For zero magnetic field or non-conducting fluid, the design reduces to an observer for the Navier-Stokes Poiseuille flow, a benchmark for flow control and turbulence estimation. The observer design for non-discretized 3-D MHD or NavierStokes channel flow has so far been an open problem.
\end{abstract}

\section{INTRODUCTION}

Recent years have been marked by dramatic advances in active flow control, but developments have had little effect on conducting fluids moving in magnetic fields. There are some recent results in stabilization though, for instance using nonlinear model reduction [4], open-loop control [8] and optimal control [12]. Other applications have been considered, for example mixing enhancement for cooling systems [20]. Some experimental results are available, showing that control of such flows is technologically feasible; actuators consist of magnets and electrodes [9], [19], [25]. Mathematical studies of controllability of magnetohydrodynamic flows have been done, though they do not provide explicit controllers [6], [24]. Despite being a subject of practical interest, there are no previous results in estimation of velocity and electromagnetic fields for conducting fluids.

In this paper, we consider an incompressible MHD channel flow, also known as the Hartmann flow, a benchmark model for applications such as cooling systems (computer systems, fusion reactors), hypersonic flight, propulsion and laser applications. In this flow, an electrically conducting fluid moves between parallel plates and is affected by an imposed transverse magnetic field. When a conducting fluid moves in the presence of a magnetic field, it produces an electric field due to charge separation and subsequently an electric current. The interaction between this created electric current and the imposed magnetic field originates a body

This work was supported by NSF grant number CMS-0329662.

R. Vazquez and M. Krstic are with the Department of Mechanical and Aerospace Engineering, University of California San Diego, La Jolla, CA 92093-0411.

Eugenio Schuster is with the Department of Mechanical Engineering and Mechanics, Lehigh University, Bethlehem, PA 18015-1835. force, called the Lorentz force, which acts on the fluid itself. The velocity and electromagnetic fields are mathematically described by the MHD equations [18], which are the NavierStokes equation coupled with the Maxwell equations.

Our observer obtains an estimate of the whole velocity, pressure, electric potential and current fields, derived only from wall measurements. Obtaining such an estimate can be of interest in itself, depending on the application. For example, the absence of effective state estimators modeling turbulent fluid flows is considered one of the key obstacles to reliable, model-based weather forecasting. In other applications in which active control is needed to achieve a given engineering objective, such as drag reduction [19], designs usually assume unrealistic full state knowledge, therefore a state estimator is necessary for effective implementation.

This paper extends our previous work for estimation of the velocity field in a 2-D channel flow [28]. Our observer is designed for the continuum MHD model. The main idea of the design is to apply the dual backstepping method for observers [23] to the estimator error system; this system is similar to the Orr-Sommerfeld-Squire system of PDE's and presents the same difficulties (non-normality leading to a large transient growth mechanism [15], [21]). Thus, applying the same ideas as in [10], we use output injection not only to guarantee stability but also to decouple the system in order to prevent transients. The output injection gains can be computed solving linear hyperbolic PDEs - a much simpler task than, for instance, solving nonlinear Riccati equations. The observer needs measurements of pressure, skin friction and current at only one of the channel walls.

If the fluid is not conductive, or there is no magnetic field, the problem reduces to the Poiseuille channel flow problem and our observer design still holds. Frequently cited as a paradigm for transition to turbulence [21], the Poiseuille flow is a benchmark for flow control and turbulence estimation. There are many results in channel flow stabilization, for instance, using optimal control [14], backstepping [27], spectral decomposition/pole placement [7], [26], Lyapunov design/passivity [1], [3], or nonlinear model reduction/indomain actuation [2]. Observer designs are more scarce; apart from the continuum backstepping approach [28], previous works were in the form of an Extended Kalman Filter for the spatially discretized Navier-Stokes equations, employing high-dimensional algebraic Riccati equations for computation of observer gains [11], [13].

The paper is organized as follows. Section II introduces the governing equations of our system. The equilibrium profile is presented in Section III and the observer structure is introduced in Section IV. Section V presents the design of the output injection gains to guarantee convergence of the observer estimates. We finish the paper with some concluding 


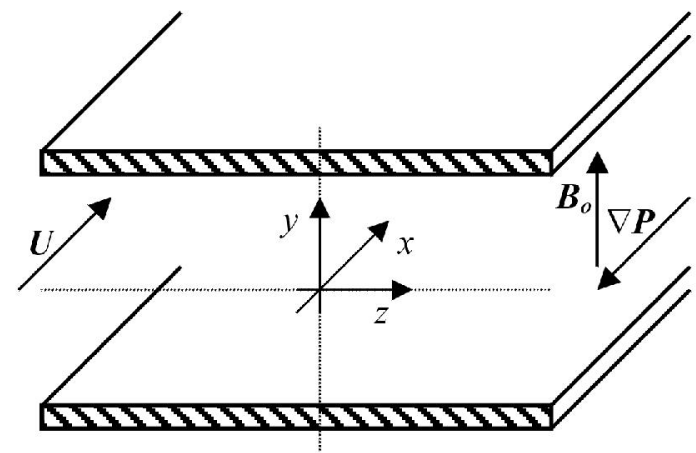

Fig, 1, Hartmann Flow.

remarks in Section VI.

\section{MODEL}

Consider an incompressible conducting fluid enclosed between two plates, separated by a distance $L$, under the influence of a pressure gradient $\nabla P$ and a magnetic field $B_{0}$ normal to the walls, as shown in Figure 1. Under the assumption of a very small magnetic Reynolds number

$$
R e_{M}=\nu \rho \sigma U_{0} L \ll 1,
$$

where $\nu$ is the viscosity of the fluid, $\rho$ the density of the fluid, $\sigma$ the conductivity of the fluid, and $U_{0}$ the reference velocity (maximum velocity of the equilibrium profile), the dynamics of the magnetic field can be neglected and the dimensionless velocity and electric potential field is governed by the inductionless MHD equations [17].

We set nondimensional coordinates $(x, y, z)$, where $x$ is the streamwise direction (parallel to pressure gradient), $y$ the wall normal direction (parallel to magnetic field), $z$ the spanwise direction, and where $(x, y, z) \in(-\infty, \infty) \times[0,1] \times$ $(-\infty, \infty)$. In these coordinates the governing equations are

$$
\begin{aligned}
U_{t}= & \frac{\triangle U}{R e}-U U_{x}-V U_{y}-W U_{z}-P_{x}+N \phi_{z} \\
& -N U, \\
V_{t}= & \frac{\triangle V}{R e}-U V_{x}-V V_{y}-W V_{z}-P_{y}, \\
W_{t}= & \frac{\triangle W}{R e}-U W_{x}-V W_{y}-W W_{z}-P_{z}-N \phi_{x} \\
& -N W, \\
\triangle \phi= & U_{z}-W_{x},
\end{aligned}
$$

where $U, V$ and $W$ denote, respectively, the streamwise, wall-normal and spanwise velocities, $P$ the pressure, $\phi$ the electric potential, $R e=\frac{U_{0} L}{\nu}$ is the Reynolds number and $N=\frac{\sigma L B_{0}^{2}}{\rho U_{0}}$ the Stuart number. Since the fluid is incompressible, the continuity equation is verified

$$
U_{x}+V_{y}+W_{z}=0 .
$$

The boundary conditions for the velocity field are

$$
\begin{aligned}
U(t, x, 0, z) & =U(t, x, 1, z)=0 \\
V(t, x, 0, z) & =V(t, x, 1, z)=0 \\
W(t, x, 0, z) & =W(t, x, 1, z)=0
\end{aligned}
$$

and assuming perfectly conducting walls, $\phi$ verifies

$$
\phi(t, x, 0, z)=\phi(t, x, 1, z)=0 .
$$

The nondimensional electric current, $j(t, x, y, z)$, is a vector field that can be directly computed from the electric potential and velocity fields as follows,

$$
\begin{aligned}
& j^{x}(t, x, y, z)=-\phi_{x}-W, \\
& j^{y}(t, x, y, z)=-\phi_{y}, \\
& j^{z}(t, x, y, z)=-\phi_{z}+U,
\end{aligned}
$$

where $j^{x}, j^{y}$, and $j^{z}$ denote the components of $j$.

Remark 1: If we set $N=0$ (zero magnetic field, or nonconducting fluid) in equations (2)-(5), they reduce to the classical Navier-Stokes equations without body forces. Then equations (2)-(4), (6) and (7)-(9) describe a pressure driven channel flow, the so-called Poiseuille flow.

\section{EQUILIBRIUM PROFILE}

The equilibrium profile for system (2)-(5) can be calculated assuming a steady solution with only one nonzero nondimensional velocity component, $U^{e}$, that depends only on the $y$ coordinate. Substituting $U^{e}$ in equation (2), one finds that it verifies the following equation,

$$
0=\frac{U_{y y}^{e}(y)}{R e}-P_{x}^{e}-N U^{e}(y),
$$

whose solution is, setting $P^{e}$ such that the maximum velocity (centerline velocity) is 1 ,

$$
\begin{aligned}
U^{e}(y) & =\frac{\sinh (H(1-y))-\sinh H+\sinh (H y)}{2 \sinh H / 2-\sinh H} \\
V^{e} & =W^{e}=\phi^{e}=0 \\
P^{e} & =\frac{N \sinh H}{2 \sinh H / 2-\sinh H} x .
\end{aligned}
$$

where $H=\sqrt{\operatorname{ReN}}=B_{0} L \sqrt{\frac{\sigma}{\rho \nu}}$ is the Hartmann number. In Fig. III(left) we show $U^{e}(y)$ for different values of $H$. Since $U^{e}(y)$ is nondimensional the centerline velocity is always 1. For $H=0$ the classic parabolic Poiseuille profile is recovered. In Fig. III(right) we show the equilibrium velocity gradient, $U_{y}^{e}(y)$, proportional to shear stress, whose maximum is reached at the boundaries and grows with $H$.

\section{OBSERVER}

Define the fluctuation variable

$$
u(t, x, y)=U(t, x, y)-U^{e}(y)
$$

where $U^{e}(y)$ is the equilibrium of the Hartmann flow, as defined in (15). The linearization of (2)-(4) around the Hartmann equilibrium profile, using (18), is

$$
\begin{aligned}
u_{t}= & \frac{\triangle u}{R e}-U^{e}(y) u_{x}-u_{y}^{e}(y) V-P_{x}+N \phi_{z} \\
& -N u, \\
V_{t}= & \frac{\triangle V}{R e}-U^{e}(y) V_{x}-P_{y}, \\
W_{t}= & \frac{\triangle W}{R e}-U^{e}(y) W_{x}-P_{z}-N \phi_{x}-N W .
\end{aligned}
$$

We design the observer for the linearized equations. It consists of a copy of (19)-(21) and (5)-(10), to which we 

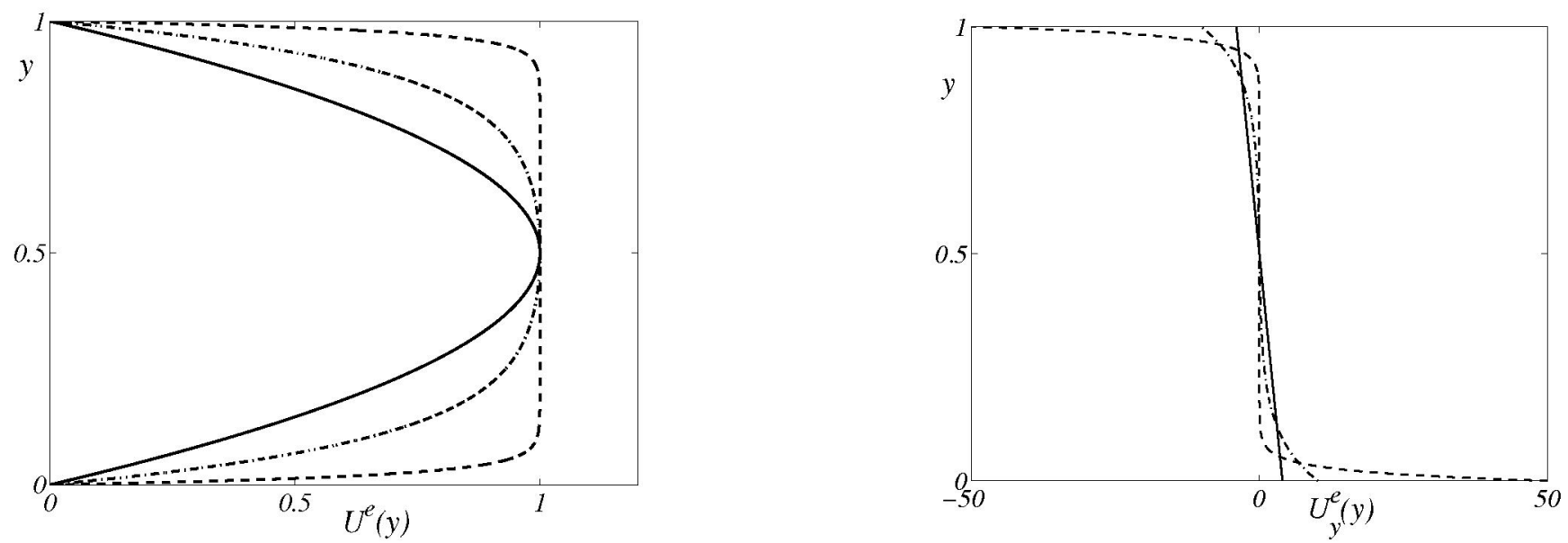

Fig. 2. Streamwise equilibrium velocity (left) and gradient of streamwise equilibrium velocity (right), for different values of $H$. Solid, $H=0$; dash-dotted, $H=10$; dashed, $H=50$.

add output injection of the pressure $P$, the potential flux $\phi_{y}$ (proportional to current), and both $u_{y}$ and $W_{y}$ (proportional to friction) at the bottom wall.

Denoting the observer (estimated) variables by a hat, the equations for the estimated velocity field are

$$
\begin{aligned}
\hat{u}_{t}= & \frac{\triangle \hat{u}}{R e}-U^{e}(y) \hat{u}_{x}-U_{y}^{e}(y) \hat{V}-\hat{P}_{x}+N \hat{\phi}_{z} \\
& -N \hat{u}-Q^{U}, \\
\hat{V}_{t}= & \frac{\triangle \hat{V}}{R e}-U^{e}(y) \hat{V}_{x}-\hat{P}_{y}-Q^{V}, \\
\hat{W}_{t}= & \frac{\triangle \hat{W}}{R e}-U^{e}(y) \hat{W}_{x}-\hat{P}_{z}-N \hat{\phi}_{x} \\
& -N \hat{W}-Q^{W} .
\end{aligned}
$$

The additional $Q$ terms in the observer equation are related to output injection and defined as follows.

$$
\begin{aligned}
\left(\begin{array}{c}
Q^{U} \\
Q^{V} \\
Q^{W}
\end{array}\right)= & \int_{-\infty}^{\infty} \int_{-\infty}^{\infty} \mathbf{L}(x-\xi, y, z-\zeta) \\
& \times\left(\begin{array}{c}
P(\xi, 0, \zeta)-\hat{P}(\xi, 0, \zeta) \\
u_{y}(\xi, 0, \zeta)-\hat{u}_{y}(\xi, 0, \zeta) \\
W_{y}(\xi, 0, \zeta)-\hat{W}_{y}(\xi, 0, \zeta) \\
\phi_{y}(\xi, 0, \zeta)-\hat{\phi}_{y}(\xi, 0, \zeta)
\end{array}\right) d \xi d \zeta,
\end{aligned}
$$

where $\mathbf{L}$ is an output injection kernel matrix designed to ensure convergence. The estimated potential is computed from

$$
\triangle \hat{\phi}=\hat{u}_{z}-\hat{W}_{x}
$$

and the observer verifies the continuity equation,

$$
\hat{u}_{x}+\hat{V}_{y}+\hat{W}_{z}=0
$$

and Dirichlet boundary conditions,

$$
\begin{aligned}
& \hat{u}(t, x, 0, z)=\hat{W}(t, x, 0, z)=\hat{V}(t, x, 0, z)=0, \\
& \hat{u}(t, x, 1, z)=\hat{W}(t, x, 1, z)=\hat{V}(t, x, 1, z)=0 \\
& \hat{\phi}(t, x, 0, z)=\hat{\phi}(t, x, 1, z)=0 .
\end{aligned}
$$

As inputs to the observer, appearing in (25), one needs measurements of pressure, skin friction and current in the

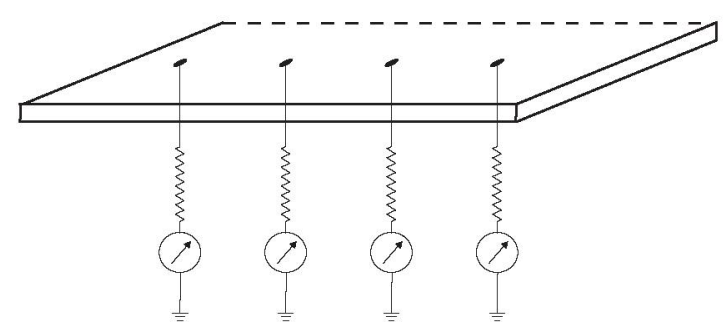

Fig. 3. An array of current sensors in the lower wall.

lower wall. For obtaining these measurements, pressure, skin friction and current sensors have to be embedded into one of the walls. Pressure and skin friction sensors are common in flow control, while for current measurement one could use an array of discrete current sensors, as depicted in Figure 3.

\section{OBSERVER DESIGN AND CONVERGENCE ANALYSiS}

Substracting (22)-(24) from (19)-(21) we obtain the error equations, with states $\tilde{U}=u-\hat{u}=U-\hat{U}, \tilde{V}=V-\hat{V}, \tilde{W}=$ $W-\hat{W}, \tilde{P}=P-\hat{P}, \tilde{\phi}=\phi-\hat{\phi}$,

$$
\begin{aligned}
\tilde{U}_{t}= & \frac{\triangle \tilde{U}}{R e}-U^{e}(y) \tilde{U}_{x}-U_{y}^{e}(y) \tilde{V}-\tilde{P}_{x}+N \tilde{\phi}_{z} \\
& -N \tilde{U}+Q^{U}, \\
\tilde{V}_{t}= & \frac{\triangle \tilde{V}}{R e}-U^{e}(y) \tilde{V}_{x}-\tilde{P}_{y}+Q^{V}, \\
\tilde{W}_{t}= & \frac{\triangle \tilde{W}}{R e}-U^{e}(y) \tilde{W}_{x}-\tilde{P}_{z}-N \tilde{\phi}_{x}-N \tilde{W}+Q^{W} .
\end{aligned}
$$

The observer error verifies the continuity equation,

$$
\tilde{U}_{x}+\tilde{V}_{y}+\tilde{W}_{z}=0
$$

while the potential error is governed by

$$
\triangle \tilde{\phi}=\tilde{U}_{z}-\tilde{W}_{x}
$$

The boundary conditions for the error states are

$$
\begin{aligned}
\tilde{U}(t, x, 0, z) & =\tilde{V}(t, x, 0, z)=\tilde{W}(t, x, 0, z)=0 \\
\tilde{U}(t, x, 1, z) & =\tilde{V}(t, x, 1, z)=\tilde{W}(t, x, 1, z)=0 \\
\tilde{\phi}(t, x, 0, z) & =\tilde{\phi}(t, x, 1, z)=0
\end{aligned}
$$


To guarantee observer convergence, our design task is to design the output injection gains $\mathbf{L}$ defined in (25) so that the origin of the error system is exponentially stable.

Since the observer error plant is linear and spatially invariant [5], we use a Fourier transform in the $x$ and $z$ coordinates (the spatially invariant directions). The transform pair (direct and inverse transform) is defined as

$$
\begin{aligned}
f\left(k_{x}, y, k_{z}\right) & =\int_{-\infty}^{\infty} \int_{-\infty}^{\infty} f(x, y, z) \mathrm{e}^{-2 \pi i\left(k_{x} x+k_{z} z\right)} d z d x, \\
f(x, y, z) & =\int_{-\infty}^{\infty} \int_{-\infty}^{\infty} f\left(k_{x}, y, k_{z}\right) \mathrm{e}^{2 \pi i\left(k_{x} x+k_{z} z\right)} d k_{z} d k_{x} .
\end{aligned}
$$

Note that we use the same symbol $f$ for both the original $f(x, y, z)$ and the image $f\left(k_{x}, y, k_{z}\right)$. In hydrodynamics $k_{x}$ and $k_{z}$ are referred to as the "wave numbers."

The observer error equations in Fourier space are

$$
\begin{aligned}
\tilde{U}_{t}= & \frac{-\alpha^{2} \tilde{U}+\tilde{U}_{y y}}{\operatorname{Re}-\beta \tilde{U}-U_{y}^{e} \tilde{V}-2 \pi k_{x} i \tilde{P}} \\
& +L^{U P} P_{0}+L^{U U} U_{y 0}+L^{U W} W_{y 0}+L^{U \phi} \phi_{y 0} \\
& +2 \pi k_{z} i N \tilde{\phi}-N \tilde{U} \\
\tilde{V}_{t}= & \frac{-\alpha^{2} \tilde{V}+\tilde{V}_{y y}}{R e}-\beta \tilde{V}-\tilde{P}_{y}+L^{V P} P_{0} \\
& +L^{V U} U_{y 0}+L^{V W} W_{y 0}+L^{V \phi} \phi_{y 0} \\
\tilde{W}_{t}= & \frac{-\alpha^{2} \tilde{W}+W_{y y}}{\operatorname{Re}-\beta \tilde{W}-2 \pi k_{z} i \tilde{P}} \\
& +L^{W P} P_{0}+L^{W U} U_{y 0}+L^{W W} W_{y 0}+L^{W \phi} \phi_{y 0} \\
& -2 \pi k_{x} i N \tilde{\phi}-N \tilde{W}
\end{aligned}
$$

where $\alpha^{2}=4 \pi^{2}\left(k_{x}^{2}+k_{z}^{2}\right), \beta=2 \pi i k_{x} U^{e}$, the $L$ 's are the entries of $\mathbf{L}$ in Fourier space (note that the convolutions in (25) become products in Fourier space). We have written for short $P_{0}=\tilde{P}\left(k_{x}, 0, k_{z}\right), U_{y 0}=\tilde{U}_{y}\left(k_{x}, 0, k_{z}\right), W_{y 0}=$ $\tilde{W}_{y}\left(k_{x}, 0, k_{z}\right), \phi_{y 0}=\tilde{\phi}_{y}\left(k_{x}, 0, k_{z}\right)$.

The continuity equation in Fourier space is expressed as

$$
2 \pi i k_{x} \tilde{U}+\tilde{V}_{y}+2 \pi k_{z} \tilde{W}=0,
$$

and the equation for the potential is

$$
-\alpha^{2} \tilde{\phi}+\hat{\phi}_{y y}=2 \pi i\left(k_{z} \tilde{U}-k_{x} \tilde{W}\right) .
$$

Note that (41)-(45) is uncoupled for each wave number. Therefore, as in [28], the range $k_{x}^{2}+k_{z}^{2} \leq M^{2}$, which we refer to as the observed wave number range, and the range $k_{x}^{2}+k_{z}^{2}>M^{2}$, the unobserved wave number range, can be studied separately. If stability for all wave numbers is established, stability in physical space follows (see [27]). The number $M$, to be computed, ensures stability for the unobserved wave number range.

We define $\chi$, a truncating function, as

$$
\chi\left(k_{x}, k_{z}\right)=\left\{\begin{array}{lc}
1, & k_{x}^{2}+k_{z}^{2} \leq M^{2} \\
0, & \text { otherwise }
\end{array}\right.
$$

Then, we reflect that we don't use output injection for unobserved wave numbers by writing

$$
\mathbf{L}=\chi\left(k_{x}, y, k_{z}\right) \mathbf{R}\left(k_{x}, y, k_{x}\right) .
$$

The matrix $\mathbf{R}$ is, like $\mathbf{L}$, defined as

$$
\mathbf{R}=\left(\begin{array}{cccc}
R^{U P} & R^{U U} & R^{U W} & R^{U \phi} \\
R^{V P} & R^{V U} & R^{V W} & R^{V \phi} \\
R^{W P} & R^{W U} & R^{W W} & R^{W \phi}
\end{array}\right)
$$

and using $\mathbf{R}$ we can write the observer error equations as

$$
\begin{aligned}
\tilde{U}_{t}= & \frac{-\alpha^{2} \tilde{U}+\tilde{U}_{y y}}{R e}-\beta \tilde{U}-U_{y}^{e} \tilde{V}-2 \pi k_{x} i \tilde{P}+\chi\left(k_{x}, k_{z}\right) \\
& \times\left\{R^{U P} P_{0}+R^{U U} U_{y 0}+R^{U W} W_{y 0}+R^{U \phi} \phi_{y 0}\right\} \\
& +2 \pi k_{z} i N \tilde{\phi}-N \tilde{U} \\
\tilde{V}_{t}= & \frac{-\alpha^{2} \tilde{V}+\tilde{V}_{y y}}{R e}-\beta \tilde{V}-\tilde{P}_{y}+\chi\left(k_{x}, k_{z}\right) \\
& \times\left\{R^{V P} P_{0}+R^{V U} U_{y 0}+R^{V W} W_{y 0} R^{V \phi} \phi_{y 0}\right\},(50) \\
\tilde{W}_{t}= & \frac{-\alpha^{2} \tilde{W}+W_{y y}}{R e}-\beta \tilde{W}-2 \pi k_{z} i \tilde{P}+\chi\left(k_{x}, k_{z}\right) \\
& \times\left\{R^{W P} P_{0}+R^{W U} U_{y 0}+R^{W W} W_{y 0}+R^{W \phi} \phi_{y 0}\right\} \\
& -2 \pi k_{x} i N \tilde{\phi}-N \tilde{W} .
\end{aligned}
$$

\section{A. Observed wave number analysis}

Consider $k_{x}^{2}+k_{z}^{2} \leq M^{2}$. Then $\chi=1$, so output injection is present. Using the continuity equation (44) and taking divergence of (49)-(51), the following Poisson equation for the pressure is derived,

$$
-\alpha^{2} \tilde{P}+\tilde{P}_{y y}=\Upsilon-4 \pi k_{x} i U_{y}^{e}(y) \tilde{V}+N V_{y},
$$

where $\Upsilon$ contains all the terms due to output injection,

$$
\begin{aligned}
\Upsilon= & P_{0}\left(2 \pi i k_{x} R^{U P}+R_{y}^{V P}+2 \pi k_{z} R^{W P}\right) \\
& +U_{y 0}\left(2 \pi i k_{x} R^{U U}+R_{y}^{V U}+2 \pi k_{z} R^{W U}\right) \\
& +W_{y 0}\left(2 \pi i k_{x} R^{U W}+R_{y}^{V W}+2 \pi k_{z} R^{W W}\right) \\
& +\phi_{y 0}\left(2 \pi i k_{x} R^{U \phi}+R_{y}^{V \phi}+2 \pi k_{z} R^{W \phi}\right)
\end{aligned}
$$

We want to make (52) independent of the output injection terms, for which we need $\Upsilon=0$. Hence, we set

$$
\begin{aligned}
R^{V P}\left(k_{x}, y, k_{z}\right)= & R^{V P}\left(k_{x}, 0, k_{z}\right)-2 \pi i \int_{0}^{y}\left(k_{x} R^{U P}\right. \\
& \left.+k_{z} R^{W P}\right)\left(k_{x}, \eta, k_{z}\right) d \eta \\
R^{V U}\left(k_{x}, y, k_{z}\right)= & R^{V U}\left(k_{x}, 0, k_{z}\right)-2 \pi i \int_{0}^{y}\left(k_{x} R^{U U}\right. \\
& \left.+k_{z} R^{W U}\right)\left(k_{x}, \eta, k_{z}\right) d \eta \\
R^{V W}\left(k_{x}, y, k_{z}\right)= & R^{V W}\left(k_{x}, 0, k_{z}\right)-2 \pi i \int_{0}^{y}\left(k_{x} R^{U W}\right. \\
& \left.+k_{z} R^{W W}\right)\left(k_{x}, \eta, k_{z}\right) d \eta \\
R^{V \phi}\left(k_{x}, y, k_{z}\right)= & R^{V \phi}\left(k_{x}, 0, k_{z}\right)-2 \pi i \int_{0}^{y}\left(k_{x} R^{U \phi}\right. \\
& \left.+k_{z} R^{W \phi}\right)\left(k_{x}, \eta, k_{z}\right) d \eta
\end{aligned}
$$

which means that, in physical space, $\nabla \cdot \mathbf{L}=0$.

Equation (52) can be solved as

$$
\begin{aligned}
\tilde{P}= & -\frac{4 \pi k_{x} i}{\alpha} \int_{0}^{y} U_{y}^{e}(\eta) \sinh (\alpha(y-\eta)) \tilde{V}\left(k_{x}, \eta, k_{z}\right) d \eta \\
& +\cosh (\alpha y) P_{0}+\frac{\sinh (\alpha y)}{\alpha} \tilde{P}_{y}\left(k_{x}, 0, k_{z}\right) \\
& +N \int_{0}^{y} \frac{\sinh (\alpha(y-\eta))}{\alpha} \tilde{V}_{y}\left(k_{x}, \eta, k_{z}\right) d \eta
\end{aligned}
$$


Evaluating equation (50) at $y=0$ one finds that

$$
\tilde{P}_{y}\left(k_{x}, 0, k_{z}\right)=\Upsilon_{0}-2 \pi i \frac{k_{x} U_{y 0}+k_{z} \tilde{W}_{y 0}}{R e},
$$

where

$$
\begin{aligned}
\Upsilon_{0}= & P_{0} R^{V P}\left(k_{x}, 0, k_{z}\right)+U_{y 0} R^{V U}\left(k_{x}, 0, k_{z}\right) \\
& +W_{y 0} R^{V W}\left(k_{x}, 0, k_{z}\right)+\phi_{y 0} R^{V \phi}\left(k_{x}, 0, k_{z}\right)(60)
\end{aligned}
$$

and again we want that the pressure be independent of the output injection terms. Hence, we set

$$
\begin{aligned}
R^{V P}\left(k_{x}, 0, k_{z}\right) & =R^{V U}\left(k_{x}, 0, k_{z}\right)=R^{V W}\left(k_{x}, 0, k_{z}\right) \\
& =R^{V \phi}\left(k_{x}, 0, k_{z}\right)=0 .
\end{aligned}
$$

Then, the pressure can be expressed as a strict-feedback [16] functional of the state $\tilde{V}$ and measurements,

$$
\begin{aligned}
\tilde{P}= & -\frac{4 \pi k_{x} i}{\alpha} \int_{0}^{y} U_{y}^{e}(\eta) \sinh (\alpha(y-\eta)) \tilde{V}\left(k_{x}, \eta, k_{z}\right) d \eta \\
& +\cosh (\alpha y) P_{0}-2 \pi i \frac{\sinh (\alpha y)}{\operatorname{Re} \alpha}\left(k_{x} U_{y 0}+k_{z} W_{y 0}\right) \\
& +N \int_{0}^{y} \frac{\sinh (\alpha(y-\eta))}{\alpha} \tilde{V}_{y}\left(k_{x}, \eta, k_{z}\right) d \eta .
\end{aligned}
$$

Similarly, solving for $\phi$ in terms of the measurement $\phi_{y 0}$ and the right hand side of its Poisson equation (45) yields

$$
\begin{aligned}
\tilde{\phi}= & \frac{2 \pi i}{\alpha} \int_{0}^{y} \sinh (\alpha(y-\eta))\left(k_{z} \tilde{U}\left(k_{x}, \eta, k_{z}\right)\right. \\
& \left.-k_{x} \tilde{W}\left(k_{x}, \eta, k_{z}\right)\right) d \eta+\frac{\sinh (\alpha y)}{\alpha} \phi_{y 0} .
\end{aligned}
$$

Introducing (62)-(63) in (49) and (51) we get

$$
\begin{aligned}
\tilde{U}_{t}= & \frac{-\alpha^{2} \tilde{U}+\tilde{U}_{y y}}{R e}-\beta \tilde{U}-U_{y}^{e}(y) \tilde{V}-N \tilde{U} \\
& +P_{0}\left(R^{U P}-2 \pi k_{x} i \cosh (\alpha y)\right) \\
& +U_{y 0}\left(R^{U U}-\frac{4 \pi^{2} k_{x}^{2}}{\alpha R e} \sinh (\alpha y)\right) \\
& +W_{y 0}\left(R^{U W}-\frac{4 \pi^{2} k_{x} k_{z}}{\alpha R e} \sinh (\alpha y)\right) \\
& +\phi_{y 0}\left(R^{U \phi}+N \frac{2 \pi k_{z} i}{\alpha} \sinh (\alpha y)\right) \\
& -\frac{8 \pi k_{x}^{2}}{\alpha} \int_{0}^{y} U_{y}^{e}(\eta) \sinh (\alpha(y-\eta)) \tilde{V}\left(k_{x}, \eta, k_{z}\right) d \eta \\
& -2 \pi i k_{x} N \int_{0}^{y} \frac{\sinh (\alpha(y-\eta))}{\alpha} \tilde{V}_{y}\left(k_{x}, \eta, k_{z}\right) d \eta \\
& -\frac{4 \pi^{2} k_{z} N}{\alpha} \int_{0}^{y} \sinh (\alpha(y-\eta)) \\
& \times\left(k_{z} \tilde{U}\left(k_{x}, \eta, k_{z}\right)-k_{x} \tilde{W}\left(k_{x}, \eta, k_{z}\right)\right) d \eta \\
\tilde{W}_{t}= & \frac{-\alpha^{2} \tilde{W}+W_{y y}-\beta \tilde{W}-N \tilde{W}}{R e} \\
& +P_{0}\left(R^{W P}-2 \pi k_{z} i \cosh (\alpha y)\right) \\
& +U_{y 0}\left(R^{W U}-\frac{4 \pi^{2} k_{x} k_{z}}{\alpha R e} \sinh (\alpha y)\right) \\
& +W_{y 0}\left(R^{W W}-\frac{4 \pi^{2} k_{z}^{2}}{\alpha R e} \sinh (\alpha y)\right)
\end{aligned}
$$

$$
\begin{aligned}
& +\phi_{y 0}\left(R^{W \phi}-N \frac{2 \pi k_{x} i}{\alpha} \sinh (\alpha y)\right) \\
& -\frac{8 \pi k_{x} k_{z}}{\alpha} \int_{0}^{y} U_{y}^{e}(\eta) \sinh (\alpha(y-\eta)) \tilde{V}\left(k_{x}, \eta, k_{z}\right) d \eta \\
& -2 \pi i k_{z} N \int_{0}^{y} \frac{\sinh (\alpha(y-\eta))}{\alpha} \tilde{V}_{y}\left(k_{x}, \eta, k_{z}\right) d \eta \\
& +\frac{4 \pi^{2} k_{x} N}{\alpha} \int_{0}^{y} \sinh (\alpha(y-\eta)) \\
& \times\left(k_{z} \tilde{U}\left(k_{x}, \eta, k_{z}\right)-k_{x} \tilde{W}\left(k_{x}, \eta, k_{z}\right)\right) d \eta
\end{aligned}
$$

Note that we have omitted the equation for $\tilde{V}$ since, from (44) and using the fact that $\tilde{V}\left(k_{x}, 0, k_{z}\right)=0, \tilde{V}$ is computed from $\tilde{U}$ and $\tilde{W}$, we have

$$
\tilde{V}=-2 \pi i \int_{0}^{y}\left(k_{x} \tilde{U}\left(k_{x}, \eta, k_{z}\right)+k_{z} \tilde{W}\left(k_{x}, \eta, k_{z}\right)\right) d \eta .
$$

We now use output injection terms to cancel the boundary terms, while still leaving some additional gains to stabilize the system. Thus, we define

$$
\begin{aligned}
R^{U P} & =2 \pi k_{x} i \cosh (\alpha y) \\
R^{W P} & =2 \pi k_{z} i \cosh (\alpha y) \\
R^{U U} & =\frac{4 \pi^{2} k_{x}^{2}}{\alpha R e} \sinh (\alpha y)+\Pi_{1}\left(k_{x}, y, k_{z}\right) \\
R^{W U} & =\frac{4 \pi^{2} k_{x} k_{z}}{\alpha R e} \sinh (\alpha y)+\Pi_{2}\left(k_{x}, y, k_{z}\right), \\
R^{U W} & =\frac{4 \pi^{2} k_{x} k_{z}}{\alpha R e} \sinh (\alpha y)+\Pi_{3}\left(k_{x}, y, k_{z}\right), \\
R^{W W} & =\frac{4 \pi^{2} k_{z}^{2}}{\alpha R e} \sinh (\alpha y)+\Pi_{4}\left(k_{x}, y, k_{z}\right), \\
R^{U \phi} & =-N \frac{2 \pi k_{z} i}{\alpha} \sinh (\alpha y), \\
R^{W \phi} & =N \frac{2 \pi k_{x} i}{\alpha} \sinh (\alpha y),
\end{aligned}
$$

where the gains $\Pi_{1}, \Pi_{2}, \Pi_{3}$ and $\Pi_{4}$ are to be defined later. From (54)-(57), (61) and (67)-(74), we get a explicit expression for the remaining entries of $\mathbf{R}$.

We introduce now the new variables

$$
Y=2 \pi i\left(k_{x} \tilde{U}+k_{z} \tilde{W}\right), \omega=2 \pi i\left(k_{z} \tilde{U}-k_{x} \tilde{W}\right) .
$$

Defining $\epsilon=\frac{1}{R e}$ and the following functions

$$
\begin{aligned}
f= & 4 \pi i k_{x}\left\{\frac{U_{y}^{e}}{2}+\int_{\eta}^{y} U_{y}^{e}(\sigma) \frac{\sinh (\alpha(y-\sigma))}{\alpha} d \sigma\right\} \\
& +N \alpha \sinh (\alpha(y-\sigma)), \\
h_{1}= & 2 \pi i k_{z} U_{y}^{e} \\
h_{2}= & -N \alpha \sinh (\alpha(y-\eta)),
\end{aligned}
$$

equations (64)-(65) expressed in terms of $Y$ and $\omega$ are

$$
\begin{aligned}
Y_{t}= & \epsilon\left(-\alpha^{2} Y+Y_{y y}\right)-\beta Y-N Y \\
& -\frac{4 \pi^{2}}{\alpha^{2}}\left(k_{x}^{2} \Pi_{1}+k_{x} k_{z} \Pi_{2}+k_{x} k_{z} \Pi_{3}+k_{z}^{2} \Pi_{4}\right) Y_{y 0} \\
& -\frac{4 \pi^{2}}{\alpha^{2}}\left(k_{x} k_{z} \Pi_{1}+k_{z}^{2} \Pi_{2}-k_{x}^{2} \Pi_{3}-k_{x} k_{z} \Pi_{4}\right) \omega_{y 0} \\
& +\int_{0}^{y} f\left(k_{x}, y, \eta, k_{z}\right) Y\left(k_{x}, \eta, k_{z}\right) d \eta,
\end{aligned}
$$




$$
\begin{aligned}
\omega_{t}= & \epsilon\left(-\alpha^{2} \omega+\omega_{y y}\right)-\beta \omega-N \omega \\
& -\frac{4 \pi^{2}}{\alpha^{2}}\left(k_{x} k_{z} \Pi_{1}-k_{x}^{2} \Pi_{2}+k_{z}^{2} \Pi_{3}-k_{x} k_{z} \Pi_{4}\right) Y_{y 0} \\
& -\frac{4 \pi^{2}}{\alpha^{2}}\left(k_{z}^{2} \Pi_{1}-k_{x} k_{z} \Pi_{2}-k_{x} k_{z} \Pi_{3}+k_{x}^{2} \Pi_{4}\right) \omega_{y 0} \\
& +h_{1}(y) \int_{0}^{y} Y\left(k_{x}, \eta, k_{z}\right) d \eta \\
& +\int_{0}^{y} h_{2}(y, \eta) \omega\left(k_{x}, \eta, k_{z}\right) d \eta
\end{aligned}
$$

where $Y_{y 0}=Y\left(k_{x}, 0, k_{z}\right)$ and $\omega_{y 0}=\omega\left(k_{x}, 0, k_{z}\right)$. We set the gains $\Pi_{1}, \Pi_{2}, \Pi_{3}$ and $\Pi_{4}$ in the following way

$$
\left(\begin{array}{c}
\Pi_{1} \\
\Pi_{2} \\
\Pi_{3} \\
\Pi_{4}
\end{array}\right)=\mathbf{A}^{-1}\left(\begin{array}{c}
l\left(k_{x}, y, 0, k_{z}\right) \\
0 \\
\theta_{1}\left(k_{x}, y, 0, k_{z}\right) \\
\theta_{2}\left(k_{x}, y, 0, k_{z}\right)
\end{array}\right) .
$$

The matrix $\mathbf{A}$ is defined as

$$
\mathbf{A}=-\frac{4 \pi^{2}}{\alpha^{2}}\left(\begin{array}{cccc}
k_{x}^{2} & k_{x} k_{z} & k_{x} k_{z} & k_{z}^{2} \\
k_{x} k_{z} & k_{z}^{2} & -k_{x}^{2} & -k_{x} k_{z} \\
k_{x} k_{z} & -k_{x}^{2} & k_{z}^{2} & -k_{x} k_{z} \\
k_{z}^{2} & -k_{x} k_{z} & -k_{x} k_{z} & k_{x}^{2}
\end{array}\right)
$$

and since $\operatorname{det}(A)=-1$ its inverse appearing in equation (81) is well-defined, whereas the functions $l\left(k_{x}, y, \eta, k_{z}\right)$, $\theta_{1}\left(k_{x}, y, \eta, k_{z}\right)$, and $\theta_{2}\left(k_{x}, y, \eta, k_{z}\right)$ in (81) are to be found. Using (81), equations (79)-(80) become

$$
\begin{aligned}
Y_{t}= & \epsilon\left(-\alpha^{2} Y+Y_{y y}\right)-\beta Y-N Y+l\left(k_{x}, y, 0, k_{z}\right) Y_{y 0} \\
& +\int_{0}^{y} f\left(k_{x}, y, \eta, k_{z}\right) Y\left(k_{x}, \eta, k_{z}\right) d \eta \\
\omega_{t}= & \epsilon\left(-\alpha^{2} \omega+\omega_{y y}\right)-\beta \omega-N \omega+\theta_{1}\left(k_{x}, y, 0, k_{z}\right) Y_{y 0} \\
& +\theta_{2}\left(k_{x}, y, 0, k_{z}\right) \omega_{y 0}+h_{1} \int_{0}^{y} Y\left(k_{x}, \eta, k_{z}\right) d \eta \\
& +\int_{0}^{y} h_{2}(y, \eta) \omega\left(k_{x}, \eta, k_{z}\right) d \eta
\end{aligned}
$$

Equations (83)-(84) are a coupled, strict-feedback plant, with integral and reaction terms. A variant of the design in [23] can be used to find $l\left(k_{x}, y, 0, k_{z}\right), \theta_{1}\left(k_{x}, y, 0, k_{z}\right)$ and $\theta_{2}\left(k_{x}, y, 0, k_{z}\right)$ using a double backstepping transformation. The transformation maps, for each $k_{x}$ and $k_{z}$, the variables $(Y, \omega)$ into the variables $(\Psi, \Omega)$, that verify the following family of heat equations (parameterized in $k_{x}, k_{z}$ ).

$$
\begin{aligned}
\Psi_{t} & =\epsilon\left(-\alpha^{2} \Psi+\Psi_{y y}\right)-\beta \Psi-N \psi, \\
\Omega_{t} & =\epsilon\left(-\alpha^{2} \Omega+\Omega_{y y}\right)-\beta \Omega-N \Omega, \\
\Psi\left(k_{x}, 0, k_{z}\right) & =\Psi\left(k_{x}, 1, k_{z}\right)=0 \\
\Omega\left(k_{x}, 0, k_{z}\right) & =\Omega\left(k_{x}, 1, k_{z}\right)=0 .
\end{aligned}
$$

The transformation is defined as follows,

$$
\begin{aligned}
Y= & \Psi-\int_{0}^{y} l\left(k_{x}, y, \eta, k_{z}\right) \Psi\left(k_{x}, \eta, k_{z}\right) d \eta \\
\omega= & \Omega-\int_{0}^{y} \theta_{1}\left(k_{x}, y, \eta, k_{z}\right) \Psi\left(k_{x}, \eta, k_{z}\right) d \eta \\
& -\int_{0}^{y} \theta_{2}\left(k_{x}, y, \eta, k_{z}\right) \Omega\left(k_{x}, \eta, k_{z}\right) d \eta .
\end{aligned}
$$

Following [22], [23], the functions $l\left(k_{x}, y, \eta, k_{z}\right)$, $\theta_{1}\left(k_{x}, y, \eta, k_{z}\right)$, and $\theta_{2}\left(k_{x}, y, \eta, k_{z}\right)$ are found as the solution of the following partial integro-differential equations,

$$
\begin{aligned}
\epsilon l_{\eta \eta}= & \epsilon l_{y y}-(\beta(y)-\beta(\eta)) l-f \\
& +\int_{\eta}^{y} f(y, \xi) l(\xi, \eta) d \xi, \\
\epsilon \theta_{1 \eta \eta}= & \epsilon \theta_{1 y y}-(\beta(y)-\beta(\eta)) \theta_{1}(y, \eta)-h_{1} \\
& +h_{1} \int_{\eta}^{y} l(\xi, \eta) d \xi+\int_{\eta}^{y} h_{2}(y, \xi) \theta_{1}(\xi, \eta) d \xi, \\
\epsilon \theta_{2 \eta \eta}= & \epsilon \theta_{2 y y}-(\beta(y)-\beta(\eta)) \theta_{2}-h_{2} \\
& +\int_{\eta}^{y} h_{2}(y, \xi) \theta_{2}(\xi, \eta) d \xi .
\end{aligned}
$$

Equations (91)-(93) are hyperbolic partial integrodifferential equation in the region $\mathcal{T}=\{(y, \eta): 0 \leq$ $y \leq 1,0 \leq \eta \leq y\}$. Their boundary conditions are

$$
\begin{aligned}
l\left(k_{x}, y, y, k_{z}\right) & =l\left(k_{x}, 1, \eta, k_{z}\right)=0, \\
\theta_{1}\left(k_{x}, y, y, k_{z}\right) & =\theta_{1}\left(k_{x}, 1, \eta, k_{z}\right)=0, \\
\theta_{2}\left(k_{x}, y, y, k_{z}\right) & ==\theta_{2}\left(k_{x}, 1, \eta, k_{z}\right)=0 .
\end{aligned}
$$

Remark 2: Equations (91)-(96) are well-posed and can be solved in several ways (see [22], [23] for techniques). Note that both Equation 91 and Equation 93 are autonomous. Hence, one must solve first for $l\left(k_{x}, y, \eta, k_{z}\right)$ and $\theta_{2}\left(k_{x}, y, \eta, k_{z}\right)$. Then the solution for $l$ is plugged into Equation 92 which then can be solved for $\theta_{1}\left(k_{x}, y, \eta, k_{z}\right)$. The observer gains are then found setting $\eta=0$ in the kernels $l\left(k_{x}, y, \eta, k_{z}\right), \theta_{2}\left(k_{x}, y, \eta, k_{z}\right)$ and $\theta_{1}\left(k_{x}, y, \eta, k_{z}\right)$.

Stability in the observed wave number range follows from stability of (85)-(86) and the invertibility of the transformation (89)-(90). The proof uses the same argument as in [22], slightly modified to account for a complex-valued kernel. See [27] for a detailed explanation.

\section{B. Unobserved wave number analysis}

When $k_{x}^{2}+k_{z}^{2}>M$, there is no output injection, as $\chi=0$. Using the change of variables (75) and following the same steps as in Section V-A, one gets the following equations for $Y$ and $\omega$ in the unobserved wave number range.

$$
\begin{aligned}
Y_{t}= & \epsilon\left(-\alpha^{2} Y+Y_{y y}\right)-\beta Y-2 \pi k_{x} i U_{y}^{e} \tilde{V}+\alpha^{2} \tilde{P} \\
& -N Y \\
\omega_{t}= & \epsilon\left(-\alpha^{2} \omega+\omega_{y y}\right)-\beta \omega-2 \pi k_{z} i U_{y}^{e} \tilde{V}-\alpha^{2} N \tilde{\phi} \\
& -N \omega .
\end{aligned}
$$

The Poisson equation for the potential is, in terms of $\omega$,

$$
-\alpha^{2} \tilde{\phi}+\phi_{y y}=\omega \text {. }
$$

Consider the Lyapunov function

$$
\begin{aligned}
\Lambda & =\int_{0}^{1} \frac{|Y|^{2}+|\omega|^{2}+\alpha^{2}|\tilde{V}|^{2}}{2} d y \\
& =\alpha^{2} \int_{0}^{1} \frac{|\tilde{U}|^{2}+|\tilde{V}|^{2}+|\tilde{W}|^{2}}{2} d y
\end{aligned}
$$

where we write, for short, $\int_{0}^{1} f=\int_{0}^{1} f\left(k_{x}, y, k_{z}\right) d y$. 
Denote by $f^{*}$ the complex conjugate of $f$. The time derivative of $\Lambda$ can be estimated as follows,

$$
\begin{aligned}
\dot{\Lambda}= & -2 \epsilon \alpha^{2} \Lambda-\epsilon \int_{0}^{1}\left(\left|Y_{y}\right|^{2}+\left|\omega_{y}\right|^{2}+\alpha^{2}\left|\tilde{V}_{y}\right|^{2}\right) \\
& -N \int_{0}^{1}\left(|Y|^{2}+|\omega|^{2}\right)-\alpha^{2} N \int_{0}^{1} \frac{\tilde{\phi}^{*} \omega+\tilde{\phi} \omega^{*}}{2} \\
& +\int_{0}^{1} \pi i U_{y}^{e}(y) \tilde{V}^{*}\left(k_{x} Y+k_{z} \omega\right) \\
& -\int_{0}^{1} \pi i U_{y}^{e}(y) \tilde{V}\left(k_{x} Y^{*}+k_{z} \omega^{*}\right) \\
& +\alpha^{2} \int_{0}^{1} \frac{P^{*} Y+P Y^{*}-P_{y}^{*} \tilde{V}-P_{y} \tilde{V}^{*}}{2}
\end{aligned}
$$

For bounding (102), we use the following two lemmas. Lemma 5.1:

$$
-\alpha^{2} \int_{0}^{1} \frac{\tilde{\phi}^{*} \omega+\tilde{\phi} \omega^{*}}{2} \leq \int_{0}^{1}|\omega|^{2}
$$

Proof: The term we want to estimate is

$$
-\alpha^{2} \int_{0}^{1} \frac{\tilde{\phi}^{*} \omega+\tilde{\phi} \omega^{*}}{2}
$$

Substituting $\alpha^{2} \phi$ from (99), (104) can be written as

$$
-\int_{0}^{1} \frac{\tilde{\phi}_{y y}^{*} \omega+\tilde{\phi}_{y y} \omega^{*}}{2}+\int_{0}^{1}|\omega|^{2}
$$

Therefore, we need to prove that

$$
\int_{0}^{1}\left(\tilde{\phi}_{y y}^{*} \omega+\tilde{\phi}_{y y} \omega^{*}\right) \geq 0 .
$$

Substituting $\omega$ from equation (99) into (106), we get

$$
\begin{aligned}
& \int_{0}^{1}\left(\tilde{\phi}_{y y}^{*} \omega+\tilde{\phi}_{y y} \omega^{*}\right) \\
= & \int_{0}^{1}\left|\tilde{\phi}_{y y}\right|^{2}-\alpha^{2} \int_{0}^{1}\left(\tilde{\phi}_{y y}^{*} \tilde{\phi}+\tilde{\phi}_{y y} \tilde{\phi}^{*}\right) \\
= & \int_{0}^{1}\left|\tilde{\phi}_{y y}\right|^{2}+\alpha^{2} \int_{0}^{1}\left|\tilde{\phi}_{y}\right|^{2}
\end{aligned}
$$

which is nonnegative.

Lemma 5.2:

$$
\left|U_{y}^{e}(y)\right| \leq 4+H
$$

Proof: Computing $U_{y}^{e}(y)$ from (15),

$$
U_{y}^{e}(y)=H \frac{\cosh (H y)-\cosh (H(1-y))}{2 \sinh H / 2-\sinh H} .
$$

Calling $g_{1}(y)=\cosh (H y)-\cosh (H(1-y))$, since $g_{1}^{\prime}(y)=$ $H(\sinh (H y)+\sinh (H(1-y)))$ is always positive for $y \in$ $(0,1)$, the maximum must be in the boundaries. Therefore

$$
\left|U_{y}^{e}(y)\right| \leq g_{2}(H)=H \frac{\cosh H-1}{\sinh H-2 \sinh H / 2} .
$$

One can rewrite $g_{2}$ as

$$
g_{2}=H \frac{\sinh H / 2}{\cosh H / 2-1} .
$$

Since $g_{2}(0)=4$, it suffices to verify that $g_{2}^{\prime}(H) \leq 1$.

$$
g_{2}^{\prime}(H)=\frac{g_{3}}{g_{4}}=\frac{\sinh H / 2-H^{2} / 2}{\cosh H / 2-1} .
$$

We need $g_{3} \leq g_{4}$. Since $g_{3}(0)=g_{4}(0)=0$, it is enough to prove $g_{3}^{\prime} \leq g_{4}^{\prime}$, which comes from the fact that

$$
g_{3}^{\prime}=H / 2(\cosh H / 2-2 H) \leq H / 2(\sinh H / 2)=g_{4}^{\prime},
$$

because $\cosh x-4 x \leq \sinh x$.

Integrating by parts and applying Lemma 5.1,

$$
\begin{aligned}
\dot{\Lambda} \leq & -2 \epsilon \alpha^{2} \Lambda-\epsilon \int_{0}^{1}\left(\left|Y_{y}\right|^{2}+\left|\omega_{y}\right|^{2}+\alpha^{2}\left|\tilde{V}_{y}\right|^{2}\right) \\
& +\int_{0}^{1} \pi i U_{y}^{e}(y) \tilde{V}^{*}\left(k_{x} Y+k_{z} \omega\right)-N \int_{0}^{1}|Y|^{2} \\
& -\int_{0}^{1} \pi i U_{y}^{e}(y) \tilde{V}\left(k_{x} Y^{*}+k_{z} \omega^{*}\right)
\end{aligned}
$$

Using Lemma 5.2 to bound $U_{y}^{e}$ in (114),

$$
\begin{aligned}
\dot{\Lambda} \leq & -2 \epsilon\left(1+\alpha^{2}\right) \Lambda-N \int_{0}^{1}|Y|^{2} d y \\
& +2 \pi(4+H) \int_{0}^{1}\left(|\tilde{V}|\left(\left|k_{x}\right||Y|+\left|k_{z}\right||\omega|\right) d y\right. \\
\leq & \left(4+H-2 \epsilon\left(1+\alpha^{2}\right)\right) \Lambda
\end{aligned}
$$

where we have applied Young's and Poincare's inequalities. Hence, if $\alpha^{2} \geq \frac{4-H}{2 \epsilon}$

$$
\dot{\Lambda} \leq-2 \epsilon \Lambda
$$

and stability in the unobserved wave number range follows when $k_{x}^{2}+k_{z}^{2} \geq M$ for $M$ (conservatively) chosen as

$$
M \geq \frac{1}{2 \pi} \sqrt{\frac{(H+4) R e}{2}} .
$$

\section{Main result}

As in [27], considering all wave numbers, the following result holds regarding the convergence of the observer.

Theorem 1: Consider the system (19)-(21) and (6), with boundary conditions (7)-(10), and the system (22)-(30), and suppose that both have classical solutions. Then, the $L^{2}$ norms of $\tilde{U}, \tilde{V}, \tilde{W}$ converge to zero, i.e.,

$\lim _{t \rightarrow \infty} \int_{-\infty}^{\infty} \int_{0}^{1} \int_{-\infty}^{\infty}\left(\tilde{U}^{2}(t)+\tilde{V}^{2}(t)+\tilde{W}^{2}(t)\right) d x d y d z=0$

Note that convergence of estimated pressure and electric potential follows from the convergence of velocity estimates.

Since we are dealing with a linearized version of the plant, this theorem has to be carefully interpreted when applied to the nonlinear system. For a fully developed MHD flow, with a Reynolds number possibly above the critical value but not too far above it, the observer is guaranteed to be convergent to the real velocity field, provided its initial estimates are not too far from the actual initial profile.

Remark 3: Since the true original plant is nonlinear, we can postulate a nonlinear observer. This observer will have the same structure and gains as the linearized observer, but adding the nonlinear terms. In this we follow the design philosophy of the Extended Kalman Filter, where gains 
are computed for the linearized case and then used for a nonlinear version of the observer. Only the equations for the estimated velocity field need to be modified, as follows

$$
\begin{aligned}
\hat{U}_{t}= & \frac{\Delta \hat{U}}{R e}-\hat{U} \hat{U}_{x}-\hat{V} \hat{U}_{y}-\hat{W} \hat{U}_{z}-\hat{P}_{x}+N \hat{\phi}_{z} \\
& -N \hat{U}-Q^{U}, \\
\hat{V}_{t}= & \frac{\triangle \hat{V}}{R e}-\hat{U} \hat{V}_{x}-\hat{V} \hat{V}_{y}-\hat{W} \hat{V}_{z}-\hat{P}_{y}-Q^{V},(120) \\
\hat{W}_{t}= & \frac{\triangle \hat{W}}{R e}-\hat{U} \hat{W}_{x}-\hat{V} \hat{W}_{y}-\hat{W} \hat{W}_{z}-\hat{P}_{z}-N \hat{\phi}_{x} \\
& -N \hat{W}-Q^{W} .
\end{aligned}
$$

In Equation (119)-(121), the $Q$ terms are the same as for the linear observer. Thus the linear gains are used for the nonlinear observer. This observer will produce closer estimates of the states in a larger range of initial conditions.

\section{CONCLUDING REMARKS}

The convergence result stated in Theorem 1 guarantees asymptotic convergence of the estimated states to the actual values of the linearized plant. For this to be true for the nonlinear plant, the estimates have to be initialized close enough to the real initial values and the MHD system has to stay in a neighborhood of the equilibrium at all times. Following [11], we may consider the mean turbulent profile instead of considering the exact laminar equilibrium profile. This amounts to changing $U^{e}$ in definition (15). Since $U^{e}$ appears in Equations (76)-(78), which are used to compute output injection gains in Equations (91)-(93), the observer gains will change (quantitatively) for the turbulent mean profile. Then Theorem 1 still holds and guarantees convergence of estimates under the same assumptions, meaning now that the state has to stay close enough to the mean turbulent profile at all times. The use of the nonlinear observer of Remark 3 will allow larger discrepancies between the state and the profile while still producing valid estimates.

In case that $N=0$, meaning that either there is no imposed magnetic field or the fluid is nonconducting, Equations (2)-(4) are the Navier-Stokes equations and the observer reduces to a velocity/pressure estimator for a 3D channel flow. This result can be seen as dual to the channel flow control problem, which was solved in [10] using similar tools. Some physical insight can be gained analyzing this case. In the context of hydrodynamic stability theory, the linearized observer error system written in $(Y, \omega)$ variables verify equations identical to the classical Orr-SommerfeldSquire equations. This are Equations (83)-(84) for observed wave numbers and Equations (97)-(98) for unobserved wave numbers. As in [10], we use the backstepping transformations (89)-(90) not only to stabilize (using gain $l$ ) but also to decouple the system (using gains $\theta_{1}, \theta_{2}$ ) in the small wave number range, where non-normality effects are more severe. Even if the linearized system is stable, non-normality produces large transient growths [21], which enhanced by nonlinear effects may allow the error system to go far away from the origin, producing inaccurate estimates. This warrants the use of extra gains to map the system into two uncoupled heat equations (85)-(86).

\section{REFERENCES}

[1] O. M. Aamo and M. Krstic, Flow Control by Feedback: Stabilization and Mixing, Berlin:Springer, 2002.

[2] J. Baker, A. Armaou and P.D. Christofides, "Nonlinear control of incompressible fluid flow: application to Burgers' equation and 2D channel flow," J. Math. Anal. Appl., vol. 252, pp. 230-255, 2000.

[3] A. Balogh, W.-J. Liu and M. Krstic M, "Stability enhancement by boundary control in 2D channel flow," IEEE Transactions on Automatic Control, vol, 46, pp. 1696-1711, 2001.

[4] J. Baker, A. Armaou and P.D. Christofides, "Drag reduction in incompressible channel flow using electromagnetic forcing," Proceedings of the 2000 ACC, Chicago, pp. 4269-4273, 2000.

[5] B. Bamieh, F. Paganini and M.A. Dahleh M. A. , "Distributed control of spatially-invariant systems," IEEE Trans. Automatic Control, vol 45, pp. 1091-1107, 2000.

[6] V. Barbu, C. Popa, T. Havarneanu and S.S. Sritharan, "Exact controllability of magneto-hydrodynamic equations," Communications on Pure and Applied Mathematics, vol. 56(6), pp. 732-783, 2003

[7] V. Barbu, "Stabilization of a plane channel flow by wall normal controllers" Preprint, 2006.

[8] T.W. Berger, J. Kim, C. Lee and J. Lim, "Turbulent boundary layer control utilizing the Lorentz force," Phys. Fluids, vol. 12, p. 631, 2000.

[9] K.S. Breuer and J. Park, "Actuation and control of a turbulent channel flow using Lorentz forces," Phys. Fluids, vol. 16(4), p. 897, 2004.

[10] J. Cochran, R. Vazquez and M. Krstic, "Backstepping boundary control of Navier-Stokes channel flow: a 3D extension," Proceedings of the 25th American Control Conference (ACC), Minneapolis, 2006.

[11] M. Chevalier, J. Hoepffner, T.R. Bewley and D.S. Henningson, "State estimation in wall-bounded flow systems. Part 2. Turbulent flows." Journal of Fluid Mechanics, to appear, 2006.

[12] K. Debbagh, P. Cathalifaud and C. Airiau, "Optimal and robust control of small disturbances in a channel flow with a normal magnetic field," preprint, 2006.

[13] J. Hoepffner, M. Chevalier, T.R. Bewley and D.S. Henningson, "State estimation in wall-bounded flow systems. Part 1. Perturbed laminar flows." Journal of Fluid Mechanics, vol. 534, pp. 263-294, 2005.

[14] M. Hogberg, T.R. Bewley and D.S. Henningson, "Linear feedback control and estimation of transition in plane channel flow," Journal of Fluid Mechanics, vol. 481, pp.149-175, 2003.

[15] M.R. Jovanovic and B. Bamieh, "Componentwise energy amplification in channel flows," J. Fluid Mech., vol. 543, pp. 145-183, 2005.

[16] M. Krstic, I. Kanellakopoulos and P.V. Kokotovic, Nonlinear and Adaptive Control Design, New York:Wiley, 1995.

[17] D. Lee and H. Choi, "Magnetohydrodynamic turbulent flow in a channel at low magnetic Reynolds number," Journal of Fluid Mechanics, vol. 439, pp. 367-394, 2001.

[18] U. Muller and L. Buhler, Magnetofluiddynamics in Channels and Containers, Berlin:Springer, 2001.

[19] J. Pang and K.-S. Choi, "Turbulent drag reduction by Lorentz force oscillation," Phys. Fluids, vol. 16(5), L35, 2004.

[20] E. Schuster and M. Kristic, "Inverse optimal boundary control for mixing in magnetohydrodynamic channel flows," Proceedings of the 2003 CDC, Maui, Hawaii USA, 2003.

[21] P.J. Schmid and D.S. Henningson, Stability and Transition in Shear Flows, Berlin:Springer, 2001.

[22] A. Smyshlyaev and M. Krstic, "Closed form boundary state feedbacks for a class of partial integro-differential equations," IEEE Transactions on Automatic Control, vol. 49, pp. 2185-2202, 2004.

[23] A. Smyshlyaev and M. Krstic, "Backstepping observers for parabolic PDEs," Systems and Control Letters, vol. 54, pp. 1953-1971, 2005.

[24] S.S. Sritharan, V. Barbu, T. Havarneanu and C. Popa, "Local controllability for the magnetohydrodynamic equations revisited," Advances in Differential Equations, vol. 10(5), pp. 481-504, 2005.

[25] J.-P. Thibault and L. Rossi, "Electromagnetic flow control: characteristic numbers and flow regimes of a wall-normal actuator," $J$. Phys. D: Appl. Phys., vol. 36, pp. 2559-2568, 2003.

[26] R. Triggiani, "Exponential feedback stabilization of a 2-D linearized Navier-Stokes channel flow by finite-dimensional, wall-normal boundary controllers, with arbitrarily small support," preprint, 2006

[27] R. Vazquez and M. Krstic, "A closed-form feedback controller for stabilization of linearized Navier-Stokes equations: the 2D Poisseuille flow," Proceedings of the 2005 CDC, Sevilla, Spain, 2005.

[28] R. Vazquez and M. Krstic, "A closed-form observer for the channel flow Navier-Stokes system," Proceedings of the 2005 CDC, Sevilla, Spain, 2005. 